Editorial

\title{
Study of Integrability and Exact Solutions for Nonlinear Evolution Equations
}

\author{
Weiguo Rui, ${ }^{1}$ Wen-Xiu Ma, ${ }^{2}$ Chaudry Masood Khalique, ${ }^{3}$ and Zuo-nong Zhu ${ }^{4}$ \\ ${ }^{1}$ Chongqing Normal University, Chongqing 401331, China \\ ${ }^{2}$ Department of Mathematics and Statistics, University of South Florida, Tampa, FL 33620-5700, USA \\ ${ }^{3}$ International Institute for Symmetry Analysis and Mathematical Modelling, Department of Mathematical Sciences, \\ North-West University, Mafikeng Campus, Private Bag X 2046, Mmabatho 2735, South Africa \\ ${ }^{4}$ Department of Mathematics, Shanghai Jiao Tong University, 800 Dongchuan Road, Shanghai 200240, China
}

Correspondence should be addressed to Weiguo Rui; weiguorhhu@aliyun.com

Received 2 April 2014; Accepted 2 April 2014; Published 28 April 2014

Copyright (C) 2014 Weiguo Rui et al. This is an open access article distributed under the Creative Commons Attribution License, which permits unrestricted use, distribution, and reproduction in any medium, provided the original work is properly cited.

Many problems in nonlinear science associated with mechanical, structural, aeronautical, ocean, electrical, and control systems can be summarized as solving nonlinear evolution equations which arise from important models with mathematical and physical significances. Investigating integrability and finding exact solutions to the discrete and continuous evolution equations have extensive applications in many scientific fields such as hydrodynamics, condensed matter physics, solid-state physics, nonlinear optics, neurodynamics, crystal dislocation, model of meteorology, water wave model of oceanography, and fibre-optic communication. The research methods for solving nonlinear evolution equations deal with the inverse scattering transformation, the Darboux transformation, the bilinear method and multilinear method, the classical and nonclassical Lie group approaches, the Clarkson-Kruskal direct method, the deformation mapping method, the truncated Painlevé expansion, the mixing exponential method, the function expansion method, the geometrical method, the dressing method, the bifurcation theory of planar dynamical system, the auxiliary equation method, the integral bifurcation method, and so forth. The special issue examines such topics as recent research advances based on the above methods and new investigation results on solving exact solutions. Knowledge and understanding of the integrability of system and dynamical behaviors (properties) of solutions for nonlinear evolutions have led to the development of nonlinear science and successfully explained all kinds of nonlinear dynamic phenomena appearing in many scientific fields.

This special issue contains thirteen papers. In the following, we briefly review each of the papers by highlighting the significance of the key contributions.

Two papers in our special issue are devoted to discuss the integrable Hamiltonian systems, and the Lax representations of these systems are both given although the problems of discussion and the method of application are very different. In the paper titled "Two-component super AKNS equations and their finite-dimensional integrable super Hamiltonian system" by J. Yu and J. Han, the authors constructed a two-component super AKNS system via the r-matrix Lie superalgebra and gave its Lax representation. In the paper titled "Consecutive Rosochatius deformations of the Garnier system and the Hénon-Heiles system" by B. Xia and R. Zhou, an algorithm of constructing infinitely many symplectic realizations of the generalized $\mathrm{sl}(2)$ Gaudin magnet is proposed by authors. Based on this algorithm, the consecutive Rosochatius deformations of integrable Hamiltonian systems are presented by authors. In this work, the consecutive Rosochatius deformations of the Garnier system and the Hénon-Heiles system as well as their Lax representations are obtained by authors.

Two papers in our special issue are concerned about limit solutions of long wave and breather soliton. Based on the Hirota bilinear method, the authors studied the 
coupled Higgs field equation and the Davey-Stewartson equation. Some new types of solutions such as new types of doubly periodic standing wave solutions and new rational homoclinic and rogue wave solutions are obtained. In the paper titled "New types of doubly periodic standing wave solutions for the coupled Higgs field equation" by G.-q. Xu, the author obtained a new type of doubly periodic standing wave solutions for a coupled Higgs field equation based on the Hirota bilinear method and theta function identities. The Jacobi elliptic function expression and long wave limits of the periodic solutions are also presented by author. By selecting appropriate parameter values, the author analyzed the interaction properties of periodic-periodic waves and periodic-solitary waves by the use of some figures. In the paper titled "New rational homoclinic and rogue waves for Davey-Stewartson equation" by C. Liu et al., the authors studied the DSI and DSII equations by using the homoclinic breather limit method based on the Hirota bilinear method. A new family of homoclinic breather wave solution, rational homoclinic solution (homoclinic rogue wave) for DSI, and DSII equations are obtained by authors in this work. The results of investigation show that the rogue wave can be generated by extreme behavior of homoclinic breather wave in higher dimensional nonlinear wave equations.

Two papers in our special issue are concerned about the study of application to the bifurcation approach of dynamical system on solving nonlinear evolution equations. In the paper titled "Bifurcation approach to analysis of travelling waves in nonlocal hydrodynamic-type models" by J. Shi and J. Li, the authors studied the nonlocal hydrodynamic-type systems which are two-dimensional travelling wave systems with a five-parameter group. The authors employed the bifurcation approach of dynamical systems to investigate the bifurcations of phase portraits depending on the parameters of systems and analyze the dynamical behavior of the travelling wave solutions. The existence of peakons, compactons, and periodic cusp wave solutions is discussed. Some exact analytical solutions such as smooth bright solitary wave solution, smooth and nonsmooth dark solitary wave solution, and periodic wave solutions as well as uncountably and infinitely many breaking wave solutions are obtained by authors in this work. In the paper titled "Cusped and smooth solitons for the generalized Camassa-Holm equation on the nonzero constant pedestal" by D. Li et al., the authors investigated the traveling solitary wave solutions of the generalized CamassaHolm equation by using the bifurcation approach of dynamical systems. Their investigation shows that the generalized Camassa-Holm equation with nonzero constant boundary has cusped and smooth soliton solutions. Mathematical analysis and numerical simulations are provided for the soliton solutions of the generalized Camassa-Holm equation in this work.

Three papers in our special issue are devoted to discuss travelling wave solutions of mathematical physical models which are high-order and high-dimensional nonlinear evolution equations. In the paper titled "Exact solutions of a high-order nonlinear wave equation of Korteweg-de Vries type under newly solvable conditions" by W. Rui, the author studied a water wave model, a high-order nonlinear wave equation of $\mathrm{KdV}$ type under some newly solvable conditions by using the integral bifurcation method together with factoring technique. Based on his previous research works, some exact traveling wave solutions such as broken-soliton solutions, periodic wave solutions of blow-up type, smooth solitary wave solutions, and nonsmooth peakon solutions within more extensive parameter ranges are obtained by author. In particular, a series of smooth solitary wave solutions and nonsmooth peakon solutions are obtained in this work. In the paper titled "Traveling wave solutions and infinite-dimensional linear spaces of multiwave solutions to Jimbo-Miwa equation" by L. Zhang and C. M. Khalique, the authors investigated the traveling wave solutions and multiwave solutions to the $(3+1)$-Jimbo-Miwa equation. As a result, besides the exact bounded solitary wave solutions, the authors obtained the existence of two families of bounded periodic traveling wave solutions and their implicit formulas by analysis of phase portrait of the corresponding traveling wave system. The authors derived the exact 2-wave solutions and two families of arbitrary finite $\mathrm{N}$-wave solutions by studying the linear space of their Hirota bilinear equation and confirmed that the $(3+$ 1)-Jimbo-Miwa equation admits multiwave solutions of any order and is completely integrable in this work. In the paper titled "Traveling wave solution in a diffusive predator-prey system with Holling type-IV functional response" by D. Yang et al., the authors investigated the traveling wave solutions of a diffusive predator-prey system with Holling type-IV functional response. In this work, a variant of Wazewski's theorem is introduced and the extended methods are used to prove that the results are the shooting argument and the invariant manifold. The existence of traveling wave solution of the reaction-diffusion predator-prey system with Holling type-IV functional response is discussed.

The classical symmetry theory for studying differential equations is presented firstly by Lie, which has been universally used and proved to be very effective in similarity reductions and group classifications. Two papers in our special issue are devoted to discuss the Bäcklund symmetry and the Bäcklund transformation for two nonlinear evolution equations. In the paper titled "Conditional Lie-Bäcklund symmetries and reductions of the nonlinear diffusion equations with source" by J. Song et al., the conditional Lie-Bäcklund symmetry approach is used to study the invariant subspace of the nonlinear diffusion equations in this work. The authors obtained a complete list of canonical forms for such equations admitting multidimensional invariant subspaces determined by higher order conditional Lie-Bäcklund symmetries. In the paper titled "On differential equations derived from the pseudo-spherical surfaces" by $\mathrm{H}$. Yang et al., the authors constructed two metric tensor fields, by means of these metric tensor fields, the sinh-Gordon equation and the elliptic sinh-Gordon equation are obtained, which describe pseudospherical surfaces of constant negative Riemann curvature scalar. By employing the Bäcklund transformation, nonlinear superposition formulas of the sinh-Gordon equation and the elliptic sinh-Gordon equation are derived, and various new exact solutions of the equations are obtained by authors in this work. 
Two papers in our special issue are concerned about the study of application to the symbolic computation method and special function. In the paper titled "New nonlinear systems admitting Virasoro-type symmetry algebra and groupinvariant solutions" by L. Wang et al., the authors extend the application of Virasoro-type symmetry prolongation method to coupled systems with two-component nonlinear equations by using symbolic computation method. New nonlinear systems admitting infinitely dimensional centerless Virasorotype symmetry algebra are constructed by authors. Some group-invariant solutions are presented by authors in this work. In the paper titled "Elliptic travelling wave solutions to a generalized Boussinesq equation" by A. El Achab, the author investigated the different kinds of travelling wave solutions for the generalized Boussinesq wave equation by using the Weierstrass elliptic function method. As a result, some previously known solutions are recovered by author in this work.

Of course, the selected topics and papers do not provide an exhaustive study of all areas of this special issue. Nonetheless, they represent the rich and many-faceted knowledge that we have the pleasure of sharing with the readers.

\section{Acknowledgments}

The Guest Editors of this special issue would like to express appreciation to the authors for their excellent contributions and patience in assisting them. The hard work of all reviewers on these papers is also very greatly acknowledged.

Weiguo Rui

Wеп-Хіи Мa

Chaudry Masood Khalique

Zuo-nong Zhu 


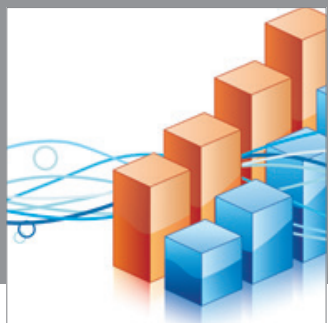

Advances in

Operations Research

mansans

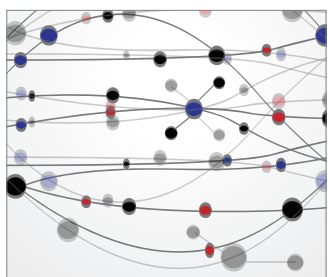

The Scientific World Journal
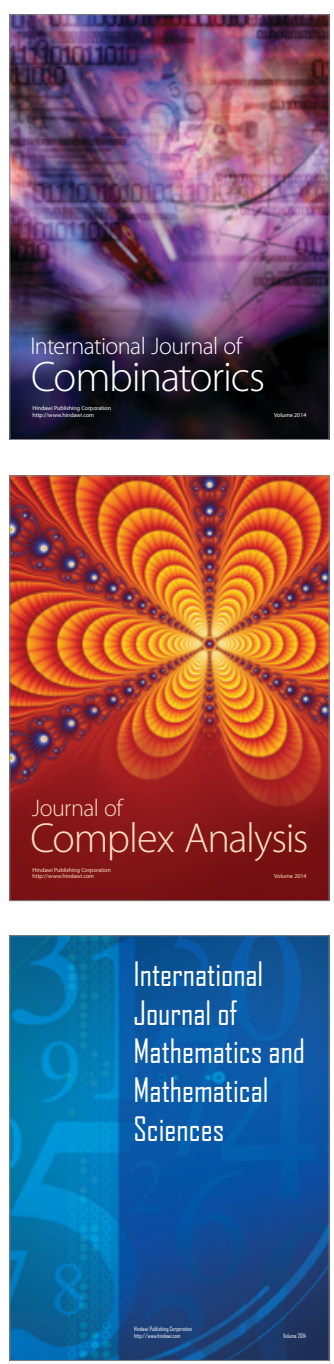
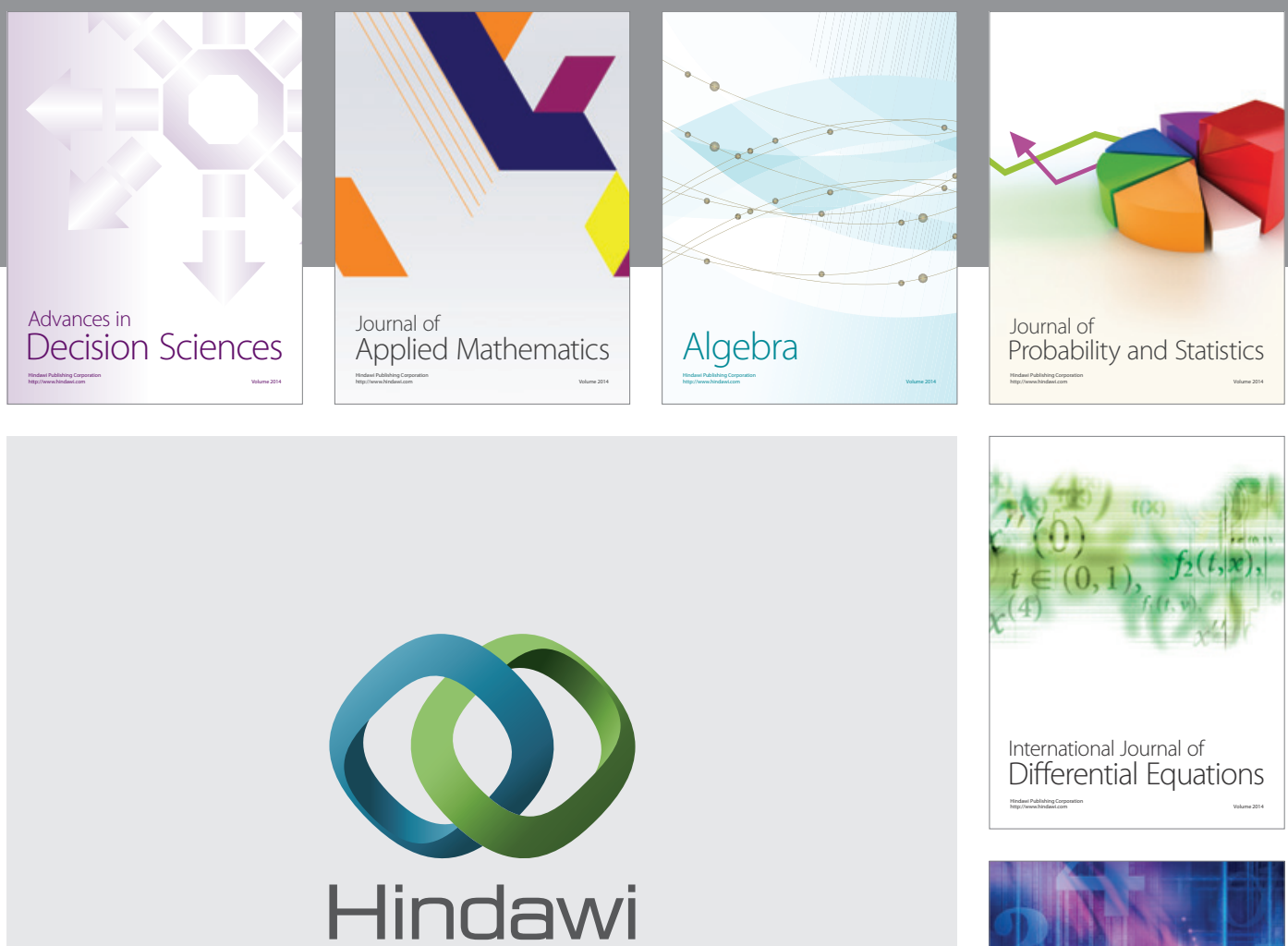

Submit your manuscripts at http://www.hindawi.com
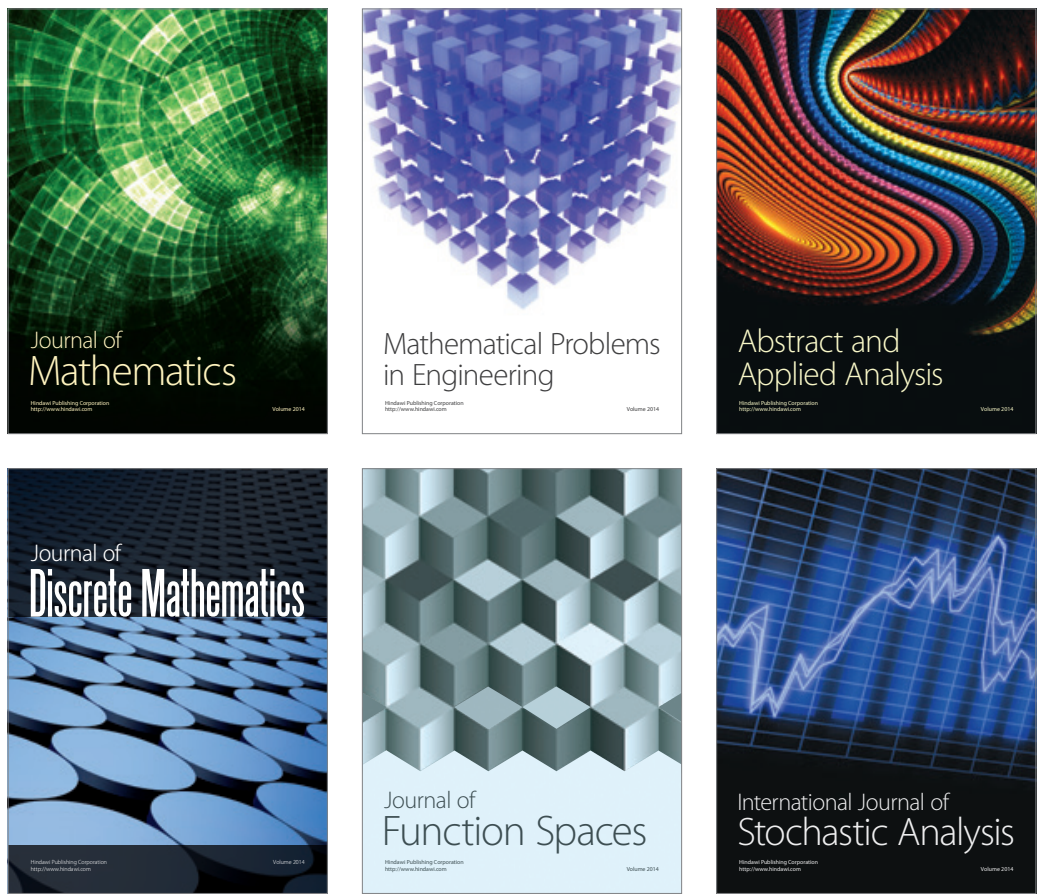

Journal of

Function Spaces

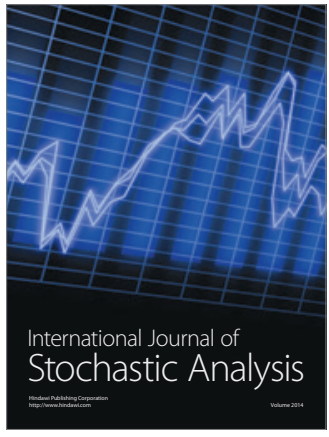

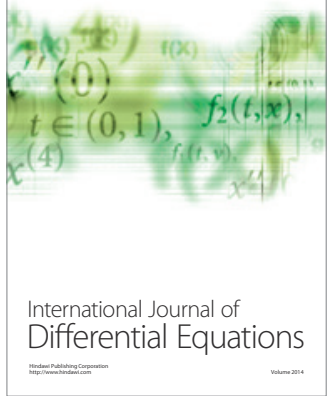
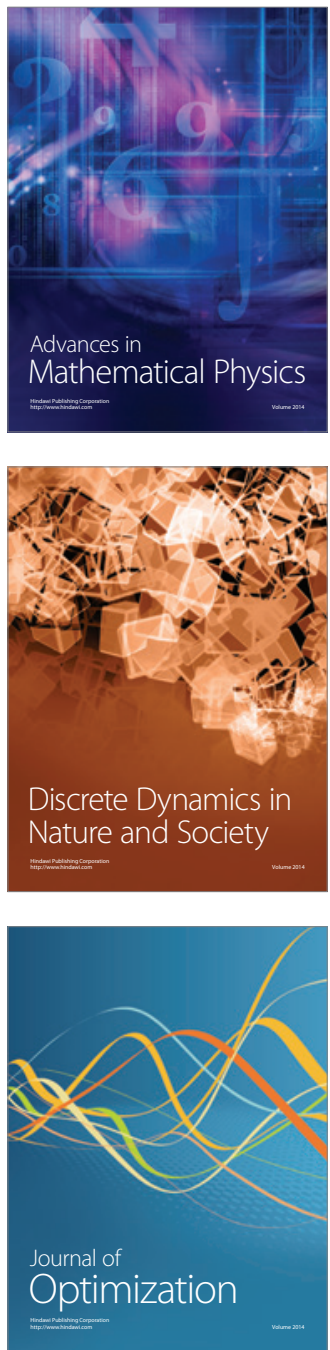